\title{
Fundamentals of Green Communications and Computing: Modeling and Simulation
}

\author{
Murat Kocaoglu, Student Member, IEEE, Derya Malak, Student Member, IEEE \\ and Ozgur B. Akan, Senior Member, IEEE
}

\begin{abstract}
Recently, ICT community has focused on developing methods to reduce energy dissipation. Although there are numerous attempts to reduce the energy dissipation of communication and computing systems in-use today, the effort to achieve fundamental energy limits for these systems is fade. In this article, we reveal the existing studies on fundamental communication and computing limits, and possible research directions. Moreover, we indicate the substantial role of ICT on carbon reduction, rather than energy. Lastly, we present efforts to find the minimum energy consumption in networks and open issues in a layered internet architecture with fundamental energy consumption per information bit.
\end{abstract}

Index Terms-green communications, green computing, fundamental limits, energy efficiency

\section{INTRODUCTION}

Greenhouse gas emissions is causing global warming and climate change. As the effects of carbon dioxide emissions have become more apparent, the ICT community has raised awareness on the subject. With the efforts of communication and computer societies, the overall carbon footprint can be reduced substantially. Consequently, the ICT community is now more concerned with diminishing adverse environmental effects of communication systems by reducing the energy consumed. Several researchers have been working on novel techniques to reduce energy dissipation of point-to-point communication links and computer networks.However, modifying the existing ICT systems could only lead us to incremental energy savings. Although not widely studied by the ICT community, fundamental energy limits for communications and computing dates back several decades. A bottom-up approach that achieves minimum energy dissipation, i.e., the fundamental energy dissipation limit, could show us how far we stand to reach

The authors are with the Next-generation and Wireless Communications Laboratory (NWCL), Department of Electrical and Computer Engineering, Koc University, Istanbul, Turkey.

Manuscript received March 12, 2012. optimum energy savings and lead with more elementary techniques to achieve the fundamental limits. Another perspective that has long been ignored by the ICT community is that, the primary purpose of reducing energy dissipation is to reduce the $\mathrm{CO}_{2}$ emission rate. It has been assumed that energy savings will provide reduced carbon emission. This hypothesis inherently presumes a linear relation between the amounts of emitted $\mathrm{CO}_{2}$ and dissipated energy. However, there might be scenarios in which optimum $\mathrm{CO}_{2}$ savings are not obtained by minimizing energy consumption. We present a non-trivial scenario in the following sections, in which minimizing the total energy consumption of links over all possible routes in a network does not correspond to minimum carbon emission. If a non-trivial dependence is observed in a more general setting, realization of ICT systems with minimum $\mathrm{CO}_{2}$ emissions will be possible, so that the Earth's atmosphere will be disturbed minimally.

The fundamental limit derived for point-to-point communications is applicable in a limited number of scenarios. It is a challenge to reveal the limits of energy dissipation in multi-terminal settings. We summarize the existing information theoretic energy limits on multiterminal communication systems. Later, due to impracticality of information theoretic approaches for large scale networks, we propose using layered architecture together with a physical layer operating at the fundamental limit of energy dissipation. We point out the novel challenges to be addressed to realize such a system and effects of constraints put on upper layers due to minimal energy consumption at the physical layer of communication infrastructures. Later, we present the current simulators with energy and carbon considerations, and point out the required developments. Lastly, we present standardization studies for green networks and open problems in the field.

\section{Green COMmunication Networks}

The primary concern of service providers in ICT has always been meeting the capacity and QoS demands 


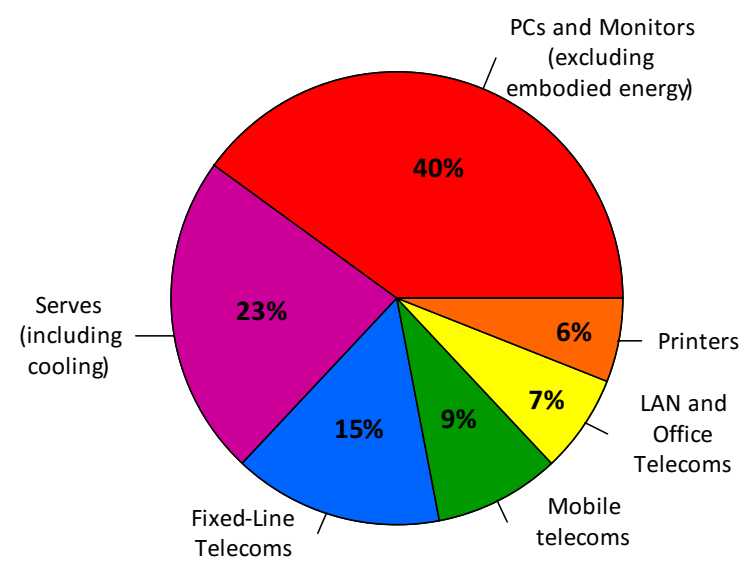

Fig. 1. Power usage of ICT devices.

of the consumers. However, in recent years, astounding increase in energy costs parallel with the increasing range of applications and equipment developed greater awareness of telecommunication industry's impact on the environment. As a result, need for reducing the carbon footprint in communications has become indisputable. Recent studies indicate that each base station antenna and its supporting equipment consume an average power of 1 Kilowatt. This amounts to a total of 8,800 Kilowatt-hours each year, which is equivalent to the energy used to run one average household in the USA [1]. The same study reports that "a medium sized network constituting 1215,000 cell sites, each equipped with 2 technologies $(2 \mathrm{G}$ \& 3G) and around 3 antennas per technology, accounts to an energy use of 736,000 Megawatt-hours, which is equivalent to running 168,000 European family homes." Green communications may mitigate excessive energy dissipation and achieve sustainable energy-efficient communications. Energy dissipation implies carbon emissions via fossil fuel-based energy sources. Therefore, green communication technologies are imperative to alleviate global warming and climate change problems.

Distinct equipments in a network have different contribution to the overall energy consumption. Fig. 1 shows the power usage of various ICT components. As observed, more than half of the energy used by ICT devices is due to communications and networking components. Therefore, significant reduction in energy consumption is possible by developing green solutions for communication networks.

Today, the green communications perception is mainly on developing energy-efficient communication techniques

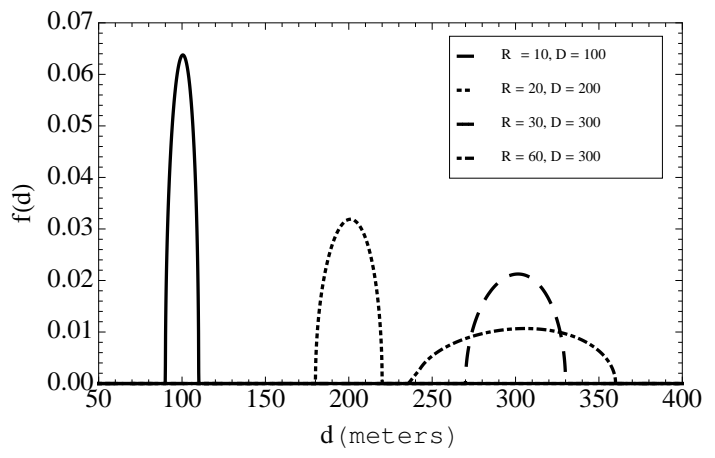

Fig. 2. Power usage of ICT devices.

for networks. 3 main approaches are suggested for power management in communication networks: Do less work, slow down and turn off idle elements. Doing less work is optimizing processes such that fewer operations are executed to reduce the energy consumption. The operation speed could be decreased to benefit from the resources efficiently. Lastly, network components and links could be shut down if not used, to reduce energy dissipation.

In [2], use of renewable energy and bio-diesel, solar/fuel powered cell sites, installation of fuel catalysts and free cooling units are suggested to reduce the carbon footprint of communications systems. Additionally, use of renewable energy sources will decrease carbon footprint without necessarily minimizing energy consumption.

However, it is not an easy task to significantly reduce the carbon footprint of ICT systems immediately, especially by only using energy efficient techniques and algorithms to improve the existing network components. As the main goal of ICT researchers has been to provide high performance and high bit rates to satisfy the growing demand, communication links and networks have been optimized to maximize the data rate, satisfying power and energy constraints. To be able to compensate the increased demand and energy consumption, ICT community should take a more revolutionary approach for developing green ICT solutions: Taking the minimization of energy as the main objective, and keeping minimum data rate and reliability as key constraints could minimize the adverse effects of ICT on the earth's atmosphere. Therefore, a need to reveal the technology-independent fundamental energy limits emerges.

A critical open issue is to incorporate the amount of $\mathrm{CO}_{2}$ emissions of ICT systems into green communications research. Furthermore, a unified study on the 
implications of fundamental limits on dynamic networks with varying rate and QoS demands is needed. In the following sections, we will present a naive snapshot of existing studies on the fundamental energy limit and also investigate the existence of a fundamental limit for $\mathrm{CO}_{2}$ emission in communications and computing. Later, we will discuss the extension of these fundamental limits to multi-terminal scenarios and point out some open issues.

\section{FUNDAMENTAL LIMITS OF ENERGY AND $\mathrm{CO}_{2}$ IN COMMUNICATIONS AND COMPUTING}

Recently, ICTs increasingly focus on saving excessive energy dissipations stemming from growing demand to relieve the adverse environmental effects on the atmosphere. In this direction, the following question arises: Is there a fundamental limit for minimum energy per bit independent from the current state of technology? Moreover, implication of such an energy limit on the existence of a fundamental $\mathrm{CO}_{2}$ emission limit is worth considering. Pointing out the fundamental limits and revealing the maximum energy savings are important to see how much we lag behind the optimum operational costs and efficiency in approaching to a greener technology.

\section{A. Fundamental Energy Limits}

The energy minimization problem has been researched from many perspectives. Some of these studies have common findings, although they have distinct starting points. The main concern is energy per bit or equivalently power per rate.

In the fundamental energy limit problem, transmission of 1 bit of information in a point to point communication link is considered. In fact, Shannon approaches this problem by a mathematical definition of information theoretic channel capacity, i.e., maximum achievable rate per channel use. From his findings, it turns out that the minimum energy required for the reliable transmission of 1 bit of information in a point to point communication link is $k T \ln 2$.

From computing perspective, the existence of one bit of information, in other words a two state system requires $k T \ln 2$ amount of energy, obtained from Boltzmann formula in the thermodynamics literature. Such a result verifies the claim that communications and computing are essentially the same processes, only with different observers in different reference frames.

Consequently, fundamental limits of communications and computing could be assumed the same, although practical considerations such as energy loss during transmission yield difference in daily use. Therefore, $k T \ln 2$ is a convenient metric to define the fundamental energy limit of communications and computing. In [3], authors define a metric using the energy per bit limit and call it the absolute energy efficiency metric given as

$$
d B \varepsilon=10 \log _{10}\left(\frac{\text { Energy } / \text { bit }}{k T \ln 2}\right) .
$$

The absolute energy efficiency metric provides a way to compare the diverse technologies. Fig. 3 presents the technologies we are currently using, capability of the human brain and efficiency of a single photon, and shows how far the state-of-the-art technology is away from the physical limits of communication.

Although the $k T \ln 2$ is a convenient physical metric for the fundamental energy limit of communications and computation of one bit of information, whether this energy needs to be dissipated or not for the transmission of information invoked discussions. In 1987, Landauer came up with the claim: He explained that, $k T \ln 2$ is the energy required to be invested to $1 \mathrm{bit}$, not to be dissipated [4]. According to Landauer, energy dissipation is required only if information is lost, which corresponds to irreversible logical operations. His claim is that, if solely logically reversible operations could be used in a computing or communicating device, dissipated energy per bit can be arbitrarily small. This idea has caused controversy among the ICT community, since it advocates no energy limit in communications.

In a recently published letter in Nature, Landauer's vision is finally verified [5]. Authors used a bead with a double-well potential to represent one bit of information and run an erasure protocol to reset the bit, by moving the bead to a predefined side of the potential well. According to the measurements, as the protocol run time spans a larger time interval, i.e., the bead's motion is slow, amount of heat dissipated approaches the Landauer's limit. It is now foreseen that, computers operating at the Landauer's limit will be realized in a few decades, yielding several orders of energy savings over current computers.

Although $k T \ln 2$ limit seems the most relevant bound on minimum energy per bit for today's ICT systems, different limits could arise in different settings and problem definitions. Shannon's derivations on channel capacity is founded on the idea of maximizing information between transmitter and receiver. $k T \ln 2$ bound is obtained as the limiting value of power per rate function, which is 


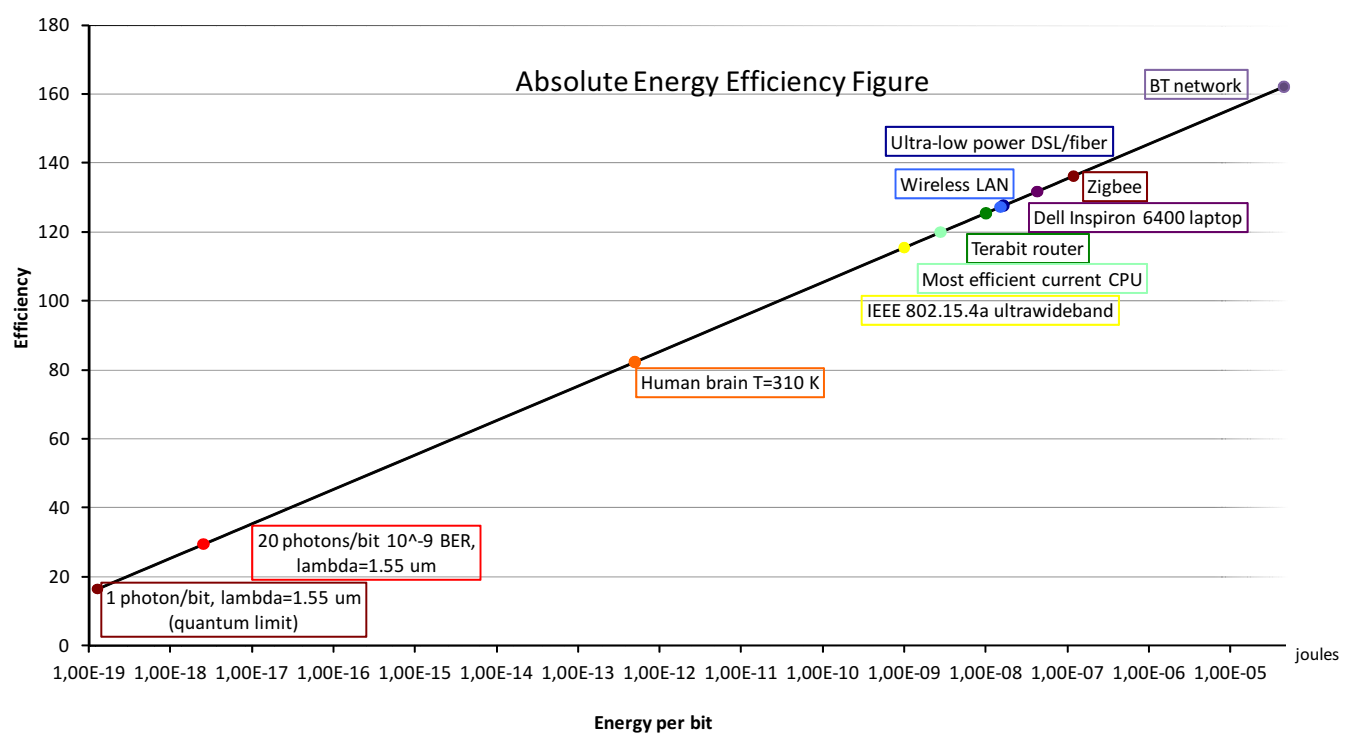

Fig. 3. Energy efficiency of state-of-the art and various limits.

obtained using Shannon's maximum information among all input distributions, i.e., capacity. A more direct investigation could aim to minimize the power per information rate expression, rather than power per channel capacity. This problem is different from minimizing inverse of the channel capacity, since the power is in the objective function, rather than being a constraint. In this way, we can understand the information rate per channel use, at which energy per bit attains a minimum value. Such an optimization problem can be defined as,

$$
\min _{p_{x}} \frac{\sigma_{x}^{2}}{I(X ; Y)},
$$

where $\sigma_{x}^{2}$ is the signal power at the receiver and $I(X ; Y)$ is the mutual information, i.e., the achievable data rate between transmitter and receiver. Minimization is over source probability distribution, i.e., $p_{x}$. Additional requirements such as satisfying certain data rates, or maximum bit error probability could be employed into such optimization problems to assure certain service quality for the end users.

\section{B. Fundamental $\mathrm{CO}_{2}$ Limits}

The main approach researchers have taken is to reduce the energy consumption for reducing the carbon footprint. This effort implicitly assumes that the emitted amount of $\mathrm{CO}_{2}$ is a linear function of the dissipated energy. Even though there is no finding suggesting a non-linear relation between energy and $\mathrm{CO}_{2}$, focusing mainly on the objective of carbon minimization rather than energy could bring new constraints into the picture. For example, efficiency of the power plants is an important factor to reduce the carbon footprint. In thermal power plants, the efficiency of power plant depends on the ratio of average load power to the peak load power. Therefore, if the power drawn from the generator is fixed over time, same amount of energy will yield less carbon emissions. For ICT systems, an open issue is to develop minimum energy achieving algorithms and techniques such that power dissipation is almost constant at all times. For example, for data modulation at the physical layer, we could prefer using binary phase shift keying, rather than on-off keying, or employ additional precautions to satisfy the fixed power constraint.

Some techniques have been proposed in the literature to reduce the carbon footprint. One of these techniques is to use energy sources with zero carbon emissions. In general, the conversion factor between energy dissipation and $\mathrm{CO}_{2}$ emission depends on the energy source. Renewable energy sources such as wind or hydroelectric energy emits no carbon during operation. However, it does not seem practical to change all the energy sources 
TABLE I

$\mathrm{CO}_{2}$ EMISSION EQUIVALENTS OF DIFFERENT ENERGY SOURCES [6].

\begin{tabular}{|c|c|c|c|}
\hline Fuel/Resource & $\begin{array}{c}\text { Thermal } \\
\text { g(CO2-eq)/MJth }\end{array}$ & $\begin{array}{c}\text { Energy Intensity } \\
\text { W.hth/W·he }\end{array}$ & $\begin{array}{c}\text { Electric } \\
\text { g(CO2-eq)/kW-he }\end{array}$ \\
\hline \multirow{2}{*}{ Coal } & $\mathrm{B}: 91.50-91.72$ & $\mathrm{~B}: 2.62-2.85$ & $\mathrm{~B}: 863-941$ \\
\cline { 2 - 4 } & $\mathrm{Br}: 94.33$ & $\mathrm{Br}: 3.46$ & $\mathrm{Br}: 1,175$ \\
\cline { 2 - 4 } & 88 & 3,01 & 955 \\
\hline $\begin{array}{c}\text { Concentrated } \\
\text { Solar Power }\end{array}$ & - & - & $40 \pm 15$ \\
\hline $\begin{array}{c}\text { Geothermal } \\
\text { Power }\end{array}$ & 3 & - & TL0-1 TH91-122 \\
\hline Hydroelectricity & - & 0,046 & 15 \\
\hline Natural gas & cc: 68.20 oc: 68.4 & - & cc: 577 oc: 751599 \\
\hline Oil & 73 & 3,4 & 893 \\
\hline Photovoltaics & - & 0,33 & 106 \\
\hline $\begin{array}{c}\text { Uranium / } \\
\text { Nuclear power }\end{array}$ & - & WL0.18 WH0.20 & WL60 WH65 \\
\hline Wind power & - & 0,066 & 21 \\
\hline
\end{tabular}

with renewable energy in short time. An alternative is to locate the network components responsible for most of the energy dissipation close to green energy sources, such as wind turbines or solar energy fields.

In Table I, common energy sources are given with their $\mathrm{CO}_{2}$ emission factors. With the $\mathrm{CO}_{2}$ equivalents of fuel sources per unit energy, total carbon footprint can be easily calculated. Consider the sample network in Fig. 4. Colors are assigned according to the carbon emission rates due to the energy source used by each node. In such a setting, in which energy sources are fixed, rate could be allocated to links so as to minimize the amount of emitted $\mathrm{CO}_{2}$. If the delay condition required by the application is satisfied, longer multi-hop paths could be used. Note that assigning carbon cost to each node and its outgoing links, we obtain a directed graph. Minimum $\mathrm{CO}_{2}$ paths are shown as continuous arrows, where others are shown with dotted arrows. Algorithms can be developed to solve a communication scenario with minimum total carbon emission. Additionally, existing cost minimization algorithms could be used for the most carbon-efficient routing scenario.

Even if whether there exists a fundamental energy limit is investigated exhaustively, fundamental $\mathrm{CO}_{2}$ limit for communications is not considered until today. Understanding fundamental $\mathrm{CO}_{2}$ limits will lead us to the optimum ICT solutions in terms of environmental awareness, even more than fundamental energy limit would.

\section{Multi-terminal extension of Fundamental LIMITS}

Achieving information theoretic fundamental energy limits is a challenging task. The theoretic minimum energy limits cannot be achieved with systems with finite bandwidth (or delay) constraints. The minimum energy per bit problem should be analyzed from a wider perspective by adding the requirements due to the finite nature of physical systems and of the communication channel, and the constraints imposed by the upper layers of the network. An open problem regarding layered architecture fundamental limits is to include the delay requirements imposed by the application layer, or the required bit error rate.

In two terminal networks, the minimum energy per bit is obtained as low SNR approximation of Shannon's capacity expression, which yields the $k_{B} T \ln 2$ limit. Extension of this approach to multi-terminal networks, i.e., the multiple-access channel, the broadcast channel, the relay channel has been the primary step for attaining the minimum energy per bit for general multi-terminal extension of energy bound. The minimum energy per bit is known for the Gaussian multiple-access, Gaussian broadcast and Gaussian interference channels [7]. However, the minimum energy per bit is still not known even for the three terminal setting of Gaussian relay channel, despite some progress [7].

In [8], the capacity per unit cost in memoryless stationary two-user and multi-terminal channels are calculated. Channel input is modeled by a cost function that associates a non-negative number to each element of the input alphabet. Author demonstrated the trade-off between the number of symbols and the cost it takes to send every unit of information through the channel.

As previously mentioned in Sec. III-B, the efficiency of power generator directly affects the total $\mathrm{CO}_{2}$ footprint. To minimize the total $\mathrm{CO}_{2}$ emissions, generator efficiency should be maximized in multi-terminal networks. Highest generator efficiency can be achieved by keeping the power drawn from the power plant constant over time. This can be done by the deployment of smart power distribution algorithms, to provide cooperation among terminals. Therefore, a centralized computing unit is inadequate to determine the optimum power allocation and scheduling in terms of carbon emissions. Hence, we could benefit from distributed computing between the network nodes, to satisfy a given power or $\mathrm{CO}_{2}$ emission requirement.

However, distributed computing might impose problems related with synchronization, parallel computing and additional feedback requirements. Furthermore, cooperation for large scale networks could cause longer delay problems as network size grows, since it gets harder to 


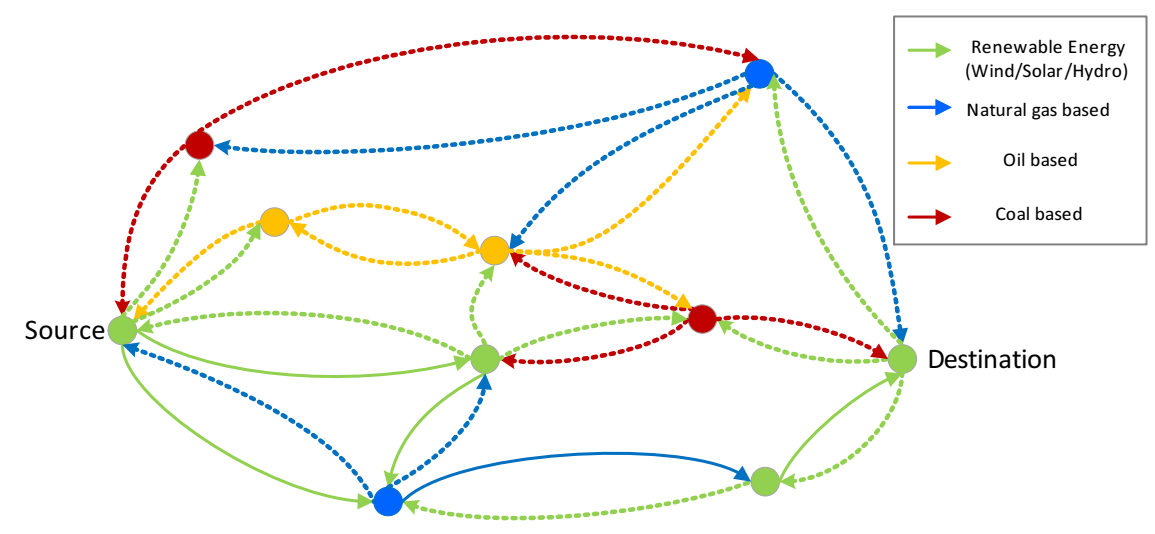

Fig. 4. Minimum $\mathrm{CO}_{2}$ path selection in a network.

obtain information from distant nodes. Therefore, allocation of distributed systems should be determined by the delay tolerance of the networks.

Today, communication mostly occurs between more than two parties concurrently over the same medium. Computer networks, building blocks of the internet as we use today, inherits many challenges over point-topoint communications. Concurrent and fair use of the finite amount of resources, addressing the desired destination with proper relay selection, flow control to provide matching data rates between transmitter and receiver are only few of the problems that arise. The idea of separating these problems to provide independent solutions led us to the current layered structure of internet. It might not be the "optimal" solution in terms of energy efficiency. However, although the information theoretic results reveal minimum energy per bit for simple settings, it is not practical to derive information theoretic solutions for large scale networks, such as worldwide web. We propose utilizing the well-known layered internet architecture together with a physical layer operating at or very close to the fundamental energy limit. Building the upper layers on top of a physical layer with minimum energy dissipation per bit, rather than minimizing energy at each layer separately is not addressed in the literature and following open issues arise in different layers.

In MAC layer, handling the channel access with minimum energy consumption is not considered. In current networks, fair channel access among multiple users is assured by either deterministic channel allocation schemes such as TDMA, FDMA, CDMA or random access techniques such as the popular CSMA schemes. If there exist many users in the network, it is not possible to allocate each user an orthogonal set of resources. Instead, a user first listens if the channel is busy. For CSMA, this is done by carrier detection. It is fair to expect that, detecting the channel state in a communication system operating at minimal energy limits should arise new challenges.

In network layer, path determination and logical addressing, and choosing the optimum path are among open issues, when physical layer operates at the minimum energy limit. Additionally, length of the addresses determine the amount of information to be processed, adding to the total energy consumption.

In transport layer, using feedback or not in flow control determines the energy dissipation and should be taken into account.

\section{Simulation of Green Networks}

Increasing number of terminals and high performance Internet demand have led to a rising trend in the network energy consumption. Therefore, as evaluation of the energy cost of large-scale infrastructures is extremely difficult, simulators greatly help us to evaluate the performance of network architectures and protocols.

There are some efforts focusing on the energy consumption of networks. An example of the simulator designs focusing on network energy consumption is a Highlevel Energy-aware Reservation Model for End-to-end networkS (HERMES) [9]. HERMES provides adaptive management of bandwidth reservations, since it coordinates the communication infrastructure by regulating traffic flows and putting the infrastructure into sleep state predictively, to save energy. HERMES is validated utilizing the Bookable Network Simulator (BoNeS) designed in Python. BoNeS generates the network topology and an 
advance bandwidth reservation traffic according to input characteristics. It simulates, with this traffic and topology, different scheduling algorithms and compares them in terms of both performance and energy consumption. 5 different scheduling schemes are run based on the generated traffic in the network and compared in terms of consumed energy. The best scheduling scheme, i.e., green scheduling, is the one yielding the minimum estimated energy consumption for each possible allocation.

Another simulation environment that could be useful in green communications research is given in [10], in which authors present a simulation environment, termed as GreenCloud, for advanced energy-aware studies of cloud computing data centers. GreenCloud is developed as an extension of the packet-level network simulator ns2. Unlike few existing cloud computing simulators such as CloudSim or MDCSim, GreenCloud extracts, aggregates, and categorizes information about the energy consumed by computing, communication elements and the physical infrastructure of a data center.

A data center scheduling methodology that combines energy efficiency and network awareness is also presented in [10]. The methodology is called data center energy-efficient network-aware scheduling (DENS). The network-awareness refers to the ability of DENS approach to receive and analyze a run-time feedback from the data center switches and links. It can also make decisions and take actions based on the network feedback, simultaneously. The DENS methodology aims to achieve the balance between individual job performances and job QoS requirements.

State-of-the-art green simulators mainly focus on predicting the best trade-offs between energy savings and traffic demand. These simulators lack incorporating the total $\mathrm{CO}_{2}$ footprint for dynamic data traffic. Suggested approach in Section III-B, i.e., the minimum $\mathrm{CO}_{2}$ emission path selection, is believed to reveal the trade-off between the energy savings and total $\mathrm{CO}_{2}$ footprint in dynamic networks. Future green network simulators should incorporate $\mathrm{CO}_{2}$ emission rates to compare network protocols in terms of environmental awareness.

\section{Standardization on Green ICT with $\mathrm{CO}_{2}$ EMISSION IN THE PICTURE}

To certify the communication systems as green, organizations and manufacturers should develop legitimate policies and standards to reduce the total carbon footprint of ICT systems.
Some international approaches assess the efficiency and the carbon footprint of telecommunication systems, and standards for industry and communications equipment [2]. The Life Cycle Assessment (LCA) is such a tool to estimate the total environmental impact of products throughout their lifespan. LCA measures GHGs and determines their $\mathrm{CO}_{2}$ equivalents. Hence, LCA determines the total carbon emissions index of the product through its lifetime; i.e., manufacture, distribution, use and disposal, including all steps beginning from the product's origin. Energy Consumption Rating (ECR) is another assessment criterion which determines the ratio of maximum energy consumption in Watts to effective system throughput in bps; i.e., the energy versus performance assessment. This technique can also be extended to the variable throughput case. There are additional tests for packetbased systems. Comparing product metrics will allow the service providers to add energy efficiency to purchase criteria.

ETSI has recently announced the second edition of a technical specification TS 102 706, which is on the measurement method for energy efficiency of wireless access equipment [11]. Energy efficiency of access networks is critical, as most of the GSM energy is dissipated in access networks. This specification standardizes the energy efficiency measurement of different wireless network technologies such as GSM/EDGE, LTE and WiMAX. Energy efficiency indicators specification considers are throughput and coverege area compared with energy consumption.

Even though many standardization efforts exist in the literature, none of them directly apply to networks operating close to the fundamental limits. A metric, similar to absolute energy efficiency metric as mentioned before, could be defined to measure the efficiency of networks and devices, compared to the fundamental energy dissipation limits. A new absolute energy efficiency metric for multi-terminal scenarios should be developed. Amount of $\mathrm{CO}_{2}$ emission should be also incorporated in any standardization effort. Such standardization together with consumer awareness will surely enforce the manufacturers to built communication and computing devices with minimum energy dissipation and $\mathrm{CO}_{2}$ emissions.

\section{CONCLUSION}

As IT solutions grow, handling the adverse environmental effects of ICT systems become more and more cumbersome. Although there are many approaches to 
reduce energy consumption of ICT systems, a fundamental approach to achieve systems with minimum energy consumption is missing. In this paper, we provide a glimpse of the existing green communication efforts and studies on fundamental energy consumption per reliable bit. Research directions that will lead us to networks operating at the fundamental energy consumption level is pointed. It is clear that, utilizing layered network architecture with physical layer operating at the fundamental energy limit is much feasible compared to information theoretical approaches, when the number of nodes in the network increases. There still exists many challenges to be addressed, before we can create green networks with minimal $\mathrm{CO}_{2}$ emissions.

\section{ACKNOWLEDGMENTS}

This work was supported in part by the TURK TELECOM under Grant Number 11315-04 and by the IBM Shared University Research Award.

\section{REFERENCES}

[1] M. Etoh, T. Ohya, and Y. Nakayama, "Energy Consumption Issues on Mobile Network Systems," International Symposium Issues on Mobile Network Systems, 2008.

[2] M. D. S. Bhawan, J. L. N. Marg, "Recommendations on Approach Towards Green Telecommunications," April 2011. [Online]. Available: http://www.trai.gov.in/Green_Telecom-12.04.2011.pdf

[3] M. Parker and S. Walker, "Roadmapping ICT: An Absolute Energy Efficiency Metric," IEEE/OSA J. Optical Commun. Netw., vol. 3, no. 8, August 2011.

[4] R. Landauer, "Energy Requirements in Communication," Applied Physics Letters, vol.51, no.24, pp.2056-2058, Dec 1987.

[5] Brut et al., "Experimental verification of Landauers principle linking information and thermodynamics," Nature, vol. 483, pp. 187189 , March 2012.

[6] "Benchmarking Energy Intensity in the Canadian Steel Industry," [Online], Available: http://oee.nrcan.gc.ca/industrial/technicalinfo/benchmarking/canadian-steel-industry/6602.

[7] A. Jain, "Minimum energy per bit for Gaussian broadcast channels with common message and cooperating receivers," 47th Annual Allerton Conference on Communication, Control, and Computing, pp.740-747, Sept. 2009.

[8] S. Verdu, "On channel capacity per unit cost," IEEE Transactions on Information Theory, , vol.36, no.5, pp.1019-1030, Sep. 1990.

[9] A.-C. Orgerie, L. Lefevre, and I. Guerin-Lassous, "Energy-Efficient Bandwidth Reservation for Bulk Data Transfers in Dedicated Wired Networks", Journal of Supercomputing, 2011.

[10] D. Kliazovich, P. Bouvry, and S. U. Khan, "A Packet-level Simulator of Energy- aware Cloud Computing Data Centers" Journal of Supercomputing, special issue on Green Networks, 2011.

[11] ETSI TS 102 706, "Environmental Engineering (EE) Measurement Method for Energy Efficiency of Wireless Access Network Equipment", Oct. 2011, [Online]. Available: http://www.etsi.org/deliver/etsi_ts/102700_102799/102706/01.02. 01_60/ts_102706v010201p.pdf
Murat Kocaoglu received his B.S degree in Electrical and Electronics Engineering with a minor degree in Physics from Middle East Technical University, Ankara, Turkey in 2010. He is now pursuing his M.S. degree in Electrical and Computer Engineering in the Next-generation and Wireless Communications Laboratory (NWCL) at Koc University. His research interests include nanocommunications, energy efficient channel and network coding.

Derya Malak received her B.Sc. degree in Electrical and Electronics Engineering from Middle East Technical University, Ankara, Turkey, in 2010. She is currently a research assistant in the Next-generation and Wireless Communications Laboratory and pursuing her M.Sc. degree at the Department of Electrical and Electronics Engineering, Koc University. Her current research interests include nanoscale communication, intra-body molecular communication networks and network information theory.

Ozgur B. Akan received B.S. and M.S. degrees in electrical and electronics engineering from Bilkent University and METU in 1999 and 2001, respectively. He received his Ph.D. degree in electrical and computer engineering from the Broadband and Wireless Networking Laboratory, School of Electrical and Computer Engineering, Georgia Institute of Technology, Atlanta, in 2004. He is a full professor with the Department of Electrical and Electronics Engineering, Koc University and director of the NWCL. His current research interests are in wireless communications, bio-inspired communications, nano-scale and molecular communications, and information theory. He is an Associate Editor of IEEE Transactions on Vehicular Technology, International Journal of Communication Systems (Wiley), and Nano Communication Networks Journal (Elsevier). He is currently General Co-Chair of ACM MOBICOM 2012, IEEE MoNaCom 2012, and TPC Co-Chair of IEEE ISCC 2012. He is a member of ACM. He received the IBM Faculty Award twice, in 2010 and 2008, and the Turkish Academy of Sciences Distinguished Young Scientist Award 2008 (TUBA-GEBIP). 